TITLE:

\title{
The association between perceived social support and amygdala structure
}

\section{$\operatorname{AUTHOR}(\mathrm{S}):$}

Sato, Wataru; Kochiyama, Takanori; Kubota, Yasutaka; Uono, Shota; Sawada, Reiko; Yoshimura, Sayaka; Toichi, Motomi

\section{CITATION:}

Sato, Wataru ...[et al]. The association between perceived social support and amygdala structure. Neuropsychologia 2016, 85: 237-244

\section{ISSUE DATE:}

2016-05-01

URL:

http://hdl.handle.net/2433/216651

\section{RIGHT:}

(c) 2016. This manuscript version is made available under the CC-BY-NC-ND 4.0 license

http://creativecommons.org/licenses/by-nc-nd/4.0/; The full-text file will be made open to the public on 1 November 2017 in accordance with publisher's 'Terms and Conditions for Self-Archiving'.; This is not the published version. Please cite only the published version.; この論文は出版社版でありません。引用の際には出版社版をご確認ご利用ください。 


\title{
The association between perceived social support and amygdala structure
}

Wataru Sato ${ }^{1, \dagger}$, Takanori Kochiyama ${ }^{2, \dagger}$, Yasutaka Kubota ${ }^{3}$, Shota Uono ${ }^{1}$, Reiko Sawada ${ }^{1}$, Sayaka Yoshimura $^{1}$, and Motomi Toichi ${ }^{4,5}$

${ }^{1}$ Department of Neurodevelopmental Psychiatry, Habilitation and Rehabilitation, Kyoto University. ${ }^{2}$ Brain Activity Imaging Center, Advanced Telecommunications Research Institute International. ${ }^{3}$ Health and Medical Services Center, Shiga University. ${ }^{4}$ Faculty of Human Health Science, Kyoto University. ${ }^{5}$ The Organization for Promoting Neurodevelopmental Disorder Research.

${ }^{\dagger}$ Equal contributors.

Corresponding author

Wataru Sato. Department of Neurodevelopmental Psychiatry, Habilitation and Rehabilitation, Graduate School of Medicine, Kyoto University, 53 Shogoin-Kawaharacho, Sakyo, Kyoto 606-8507, Japan. E-mail: sato.wataru.4v@kyoto-u.ac.jp

\begin{abstract}
The subjective perception of social support plays a crucial role in human well-being. However, its structural neural substrates remain unknown. We hypothesized that the amygdala, specifically its laterobasal and superficial subregions, which have been suggested to serve social functions, could be associated with the level of perceived social support. To test this hypothesis, we assessed perceived social support using the Multidimensional Scale of Perceived Social Support. In addition, we measured the volume and shape of the amygdala using structural magnetic resonance imaging in 49 healthy participants. Global amygdala volume in the left hemisphere was positively associated with the perceived social support score after adjusting for total cerebral volume, sex, age, intelligence, and five-factor personality domains. The local shape of the laterobasal and superficial subregions of the left amygdala showed the same association with perceived social support. These data suggest that the social subregions of the left amygdala are associated with the implementation of perceived social support.
\end{abstract}

\section{Keywords}

amygdala; laterality; magnetic resonance imaging (MRI) volumetry; Multidimensional Scale of Perceived Social Support (MSPSS); perceived social support. 


\section{Introduction}

The subjective perception of social support (i.e., the feeling of being supported by other people) plays an important role in human well-being (Lakey, 2014). Several previous psychological studies have shown that perceived social support is reliably linked to high life satisfaction, high positive affect, and low psychological distress (e.g., Diener \& Fujita, 1995; for a review, see Lakey, 2014). To investigate this important subjective construct, psychometric studies were undertaken, and several questionnaires were developed to measure perceived social support in a reliable and valid manner (e.g., Multidimensional Scale of Perceived Social Support [MSPSS]: Zimet, Dahlem, Zimet, \& Farley, 1988; for a review, see López \& Cooper, 2011). Although it intuitively appears that perceived social support is generated by actually being supported by other people, several previous studies consistently found that perceived social support was only mildly associated with receiving social support or with social network size (Barrera, 1986; Haber, Cohen, Lucas, \& Baltes, 2007; Uchino, 2009). Moreover, several psychogenetic studies evaluating twins showed that perceived social support was substantially influenced by genetic factors (Bergeman, Plomin, Pedersen, McClearn, \& Nesselroade, 1990; Kessler, Kendler, Heath, Neale, \& Eaves, 1992; Raynor, Pogue-Geile, Kamarck, McCaffery, \& Manuck, 2002) in contrast to social network size, which showed little genetic influence (Bergeman et al., 1990). Based on these data, it was proposed that perceived social support is not a state resulting from support received by a social network, but rather a stable characteristic similar to traits and personalities (Barrera, 1986; Lakey \& Lutz, 1996; Uchino, 2009).

Despite such accumulating psychological evidence for perceived social support, the neural substrate that implements perceived social support remains largely unknown. Understanding the neural substrate of perceived social support may provide an objective measure of this subjective construct, which could complement its assessment theoretically (e.g., defense against skepticism for the validity of subjective measures; Oswald \& Wu, 2010) and practically (e.g., assessment for participants with difficulty in subjective evaluation; Cummins, 2014). To date, only one previous magnetic resonance imaging (MRI) study has reported a structural neural substrate of perceived social support (Che et al., 2014a). In that study, the researchers assessed perceived social support using MSPSS (Zimet et al., 1988) and analyzed brain structures by conducting voxel-based morphometry. They found a positive association between the volume of the posterior cingulate cortex and the score of perceived social support, suggesting that this cortical region plays an important role in implementing the subjective perception of social support. However, because there are several different methods to analyze brain structures (e.g., delineation and volume measurement of the structures) with different advantages and disadvantages (Nemmi, Sabatini, Rascol, \& Peran, 2015), exploration using different analysis methods may reveal the involvement of other brain regions in perceived social support.

A candidate brain region related to perceived social support is the amygdala. Ample evidence from lesion and recording studies in humans and monkeys indicates that the amygdala plays a key role in social function (e.g., Dicks, Myers, \& Kling, 1969; for reviews, see Adolphs, 2010; Amaral, 2003; Kling \& Brothers, 1992). A previous functional MRI study also reported that the association between amygdala activation in response to negative emotional facial expressions and anxiety was reduced by 
Social support and amygdala structure 3

a higher level of perceived social support (Hyde, Gorka, Manuck, \& Hariri, 2011). Several previous structural MRI studies that investigated the structural neural substrate of social network property, which is different from but mildly related to perceived social support as described above (e.g., Barrera, 1986), found positive relationships between social network size and amygdala volume (Bickart, Wright, Dautoff, Dickerson, \& Barrett, 2011; Kanai, Bahrami, Roylance, \& Rees, 2012; Von Der Heide, Vyas, \& Olson, 2014).

No study has shown an association between amygdala structure and perceived social support. A previous study (Bickart et al., 2011) reported null findings regarding an association between amygdala volume and perceived social support using the Social Provisions Scale (Russell, Cutrona, Rose, \& Yurko, 1984). However, we postulated that different measures with different advantages used to assess perceived social support (López \& Cooper, 2011) might reveal the structural neural correlates. Whereas Che et al. (2014a) used voxel-based morphometry and did not find an association between amygdala volume and perceived social support, we postulated that a more in-depth analysis using a segmentation and measurement tool for the subcortical structures, such as the FMRIB's Integrated Registration and Segmentation Tool (FIRST) (Patenaude, Smith, Kennedy, \& Jenkinson, 2011), could detect an association more sensitively. Therefore, we used a different scale and analysis method from the previous studies, and hypothesized that amygdala volume would be positively related to perceived social support.

Furthermore, we expected that specific subregions associated with perceived social support could be identified in the amygdala. Anatomical and physiological studies in animals have provided ample evidence that the amygdala consists of structurally and functionally distinct subregions, although the number and classification of subregions remain inconclusive (for a review, see Swanson \& Petrovich, 1998). One of the dominant theories proposes that the human amygdala can be separated into three subregions: the laterobasal, superficial (cortical), and centromedial divisions (Heimer et al., 1999). Anatomical studies in animals have shown that these subregions have different cytoarchitectonic patterns in which the laterobasal and superficial subregions have cell types similar to those of the neocortex, while the centromedial subregion has neurons similar to those of the striatum (for a review, see McDonald, 1998). Animal studies also reported that these subregions have different connectivity patterns with other regions: the laterobasal-superficial subregions and the centromedial subregion comprise separate groups (for a review, see McDonald, 1998), and there are strong connections between the laterobasal group and frontotemporal system and between the centromedial subregion and autonomic system (for a review, see Swanson \& Petrovich, 1998). This classification of amygdala subregions was implemented in probability maps in stereotaxic space in humans (Amunts et al., 2005), and some functional neuroimaging studies reported that emotional facial expressions specifically activated the laterobasal (Hurlemann et al., 2008) and superficial (Goossens et al., 2009; Hurlemann et al., 2008), but not the centromedial, subregions of the amygdala. These data suggest the possibility that the laterobasal and superficial subregions, compared with the centromedial subregion, might be more involved in social functioning. Consistent with this, a stereological study indicated that individuals with autism spectrum disorders (ASD), which are characterized primarily by qualitative impairments in social interaction (American Psychiatric Association, 2013), had fewer neurons in the laterobasal and superficial subregions but not in the centromedial subregion 
Social support and amygdala structure 4

(Schumann \& Amaral, 2006). Comparative anatomical studies also showed that the laterobasal and superficial, but not the centromedial, subregions of the amygdala have enlarged in an evolutionarily ascending order in primates (Barton \& Aggleton, 2000; Stephan \& Andy, 1977), especially in humans versus non-human primates (Barger, Hanson, Teffer, Schenker-Ahmed, \& Semendeferi, 2014; Barton \& Aggleton, 2000), and hence some researchers suggested that these subregions may be related to the evolution of social functioning in primates (e.g., Barger et al., 2014). Based on these data, we hypothesized that the enlargement of the laterobasal and superficial amygdala subregions might be specifically associated with a heightened perceived social support.

To test these hypotheses, we investigated the relationship between perceived social support and the global volume and local shape of the amygdala. We assessed perceived social support using the MSPSS (Zimet et al., 1988). To measure the volume and shape of the amygdala, we acquired structural MR images and delineated the bilateral amygdala using an automated method (Patenaude et al., 2011).

\section{Materials and methods}

\subsection{Participants}

The study included 49 Japanese volunteers (23 females; mean $\pm S D$ age, $22.4 \pm 4.4$ years). A psychiatrist or psychologist administered a short structured diagnostic interview using the MiniInternational Neuropsychiatric Interview (Sheehan et al., 1998); no neuropsychiatric problem was detected in any participant. All participants were right-handed, as assessed by the Edinburgh Handedness Inventory (Oldfield, 1971). After the procedures were fully explained, all participants provided informed consent for participation. This study was approved by the local ethics committee of the Primate Research Institute, Kyoto University.

\subsection{Psychological questionnaires}

We used the Japanese version of the MSPSS (Zimet et al., 1988; Iwasa et al., 2007) to measure perceived social support. The questionnaire included 12 items that assessed perceived social support (e.g., "I get the emotional help and support I need from my family”). Four items comprised a subscale and measured perceived social support from three distinct sources: family, friends, and significant others (e.g., spouses; Zimet, Powell, Farley, Werkman, \& Berkoff, 1990). Each item was scored on a scale from 1 (very strongly disagree) to 7 (very strongly agree). The reliability and validity of the questionnaire were confirmed against the original (Zimet et al., 1988, 1990) and Japanese (Iwasa et al., 2007) versions.

We assessed the participants' personality domains to confirm that the results could not be accounted for by their basic personalities, not specific to perceived social support. We used the Japanese version of the NEO Five-Factor Inventory (Shimonaka et al., 1999), a 60-item self-rating questionnaire that measures the five domains of personality: neuroticism, extraversion, openness to experience, agreeableness, and conscientiousness (12 items for each) (McCrae \& Costa, 1987). The reliability and validity of the questionnaire have been verified in Japanese participants (Shimonaka et al., 1999). 
Social support and amygdala structure 5

We also assessed participants' intelligence quotients (IQs), because the subjective ratings can be confounded by intelligence (Cummins, 2014). We used the revised Wechsler Adult Intelligence Scale, third edition (Nihon Bunka Kagakusha, Tokyo, Japan).

\subsection{MRI acquisition}

Image scanning was performed on a 3-T MRI system (MAGNETOM Trio, A Tim System, Siemens) at the ATR Brain Activity Imaging Center using a 12-channel head coil. A forehead pad was used to stabilize the head position. A T1-weighted high-resolution anatomical image was obtained using a magnetization-prepared rapid-acquisition gradient-echo (MP-RAGE) sequence (repetition time $=2,250 \mathrm{~ms}$; echo time $=3.06 \mathrm{~ms}$; inversion time $=1,000$; flip angle $=9^{\circ}$; field of view $=256 \times 256 \mathrm{~mm}$; voxel size $=1 \times 1 \times 1 \mathrm{~mm}$ ).

\subsection{Psychological data analysis}

For the MSPSS, the means of all 12 items were calculated as the measure of perceived social support. For personality assessment, the total scores of all 12 items were calculated in each of the five personality domains.

\subsection{Image analysis: segmentation}

Using FIRST in the FMRIB Software Library (FSL) ver. 5 (Patenaude, 2007), segmentation and analysis of the MRI brain structures was performed. FIRST is an automated subcortical segmentation tool used for efficient modeling of the brain shape and appearance within a Bayesian framework (Patenaude et al., 2011). The method was validated (Patenaude et al., 2011) and used in a wide range of applications for volumetric analyses (e.g., Pardini, Raine, Erickson, \& Loeber, 2014; Sato et al., 2014). The analyses were conducted using standard FIRST procedures (Patenaude et al., 2011). First, the images were registered to a common space based on the Montreal Neurological Institute (MNI)152 template using affine registration of the entire head and a subcortical mask to exclude voxels outside the subcortical regions. Then, the inverse transformation was applied to bring the images back into the original space. Finally, the brain structures were segmented based on a Bayesian shape and appearance model, which is part of the FSL package, and were originally constructed from manually segmented images provided by the Centre for Morphometric Analysis (Boston, MA, USA). One of the study authors (T.K.) validated the success of the automated segmentation procedure and visually inspected the segmentation quality using a FSL-provided script to generate summary images in a well-organized webpage format (first_roi_slicesdir). The bilateral amygdala was segmented for each participant. Other subcortical structures were also segmented for comparison, including the thalamus, caudate, putamen, globus pallidus, hippocampus, and nucleus accumbens.

\subsection{Image analysis: volume analysis}

The volume of the amygdala was calculated for each hemisphere in each participant using the fslstat script provided by FSL. To control for the total cerebral volume (TCV), modulated gray and white matter images were obtained using VBM8 software (http://dbm.neuro.uni-jena.de/vbm/). The sum of the gray and white matter volumes was then calculated by integrating voxel intensities over the entire segmented image and then multiplying by voxel size.

The association between amygdala volume and perceived social support was tested using multiple regression analysis. The regression model contained the amygdala volume as the dependent variable and the perceived social support score, hemisphere, and their interaction as the effect-of-interest independent variables. Sex, age, full-scale IQ, the five domains of personality, and TCV were also 
Social support and amygdala structure 6

included as covariates (effects of no interest). Main effects or interactions related to the perceived social support factor were regarded as effects of interest. When the interaction between social support and hemisphere was significant, follow-up analyses were conducted using a general linear model without the hemisphere factor. Based on our prediction of a positive relationship between amygdala volume and perceived social support, the effect of perceived social support was tested using $t$ statistics (one-tailed). To check outliers for parametric statistics, we calculated Cook's distance and found no case with scores greater than 1.0 (Cook \& Weisberg, 1982). Although we did not have specific hypotheses, we conducted exploratory analyses of the relationships between the volumes of brain regions other than the amygdala and the MSPSS total score using the same software (Patenaude et al., 2011). The results were considered statistically significant at $p<0.05$.

\subsection{Image analysis: shape analysis}

The shape of the amygdala was analyzed by performing surface morphometric analysis (Patenaude et al., 2011) as part of the FSL software. Surface meshes were created on the left and right amygdala using a deformable mesh model. Each mesh was comprised of a set of triangles; the apexes connecting the triangles were called vertices. The number of vertices was fixed so that the corresponding vertices were comparable across participants. The projections were scalar values, which represented the vertex displacement from the average surface to each individual surface and served as the dependent variable in the following multiple regression analyses. All meshes were reconstructed in MNI space to normalize brain size. Surface meshes were reconstructed in MNI space to normalize brain size; this process consequently controlled the effect of TCV.

To identify changes in vertices associated with perceived social support, multiple regression analyses were conducted using perceived social support score as the independent variable and using sex, age, full-scale IQ, and the five domains of personality as covariates (effects of no interest). The relationship between the vertex change and perceived social support was assessed using a nonparametric permutation-based inference (Winkler, Ridgway, Webster, Smith, \& Nichols, 2014). We generated 5,000 permutations of the data to test against when building up the null distribution. The results were considered statistically significant at $p<0.05$ (cluster-level family-wise error-corrected) based on the threshold-free cluster enhancement method (Smith \& Nichols, 2009). Amygdala subregions were identified according to the cytoarchitectonic map derived from human postmortem brain data using Anatomy Toolbox ver. 2.0 (Amunts et al., 2005; Eickhoff et al., 2005). To assess the correspondence between global volume and local shape of the amygdala, we also calculated Pearson's product-moment correlations between global volumes and perpendicular distances at peak foci in the amygdala.

\section{Results}

\subsection{Psychological rating}

The results of psychological ratings and their Pearson's product-moment correlations are shown in Table 1. The perceived social support score (mean $\pm S D, 5.2 \pm 1.1$ ) was comparable to that in a previous study (t-test, $p>0.1$; Iwasa et al., 2007). Correlation analyses showed a significant association between the perceived social support score and sex $(r=0.32 ; p<0.05)$ with higher scores in females, as in previous studies (e.g., Zimet et al., 1988; Iwasa et al., 2007). There were no significant correlations between the perceived social support score and other variables, including age, full-scale IQ, and personality domains ( $p>0.1)$. 
Social support and amygdala structure 7

\subsection{Volume analysis}

The general linear model analysis that tested the effect of perceived social support and hemisphere on amygdala volume partialling out the effect of sex, age, full-scale IQ, the five domains of personality, and TCV revealed a significant interaction between the perceived social support score and hemisphere $(t(38)=2.98, p<0.005)$, indicating that the left and right amygdala volumes were differentially related to the level of perceived social support (Fig. 1). The main effect of perceived social support was not significant $(t(38)=0.98, p>0.1)$. Follow-up analysis of data from each hemisphere revealed that the relationship between the amygdala volume and perceived social support score was significant and positive in the left hemisphere $(t(38)=1.95, p<0.05)$, but was not significant in the right hemisphere $(t(38)=1.36, p>0.1)$.

Because the software can output the volumes of other brain structures including the thalamus, caudate, putamen, globus pallidus, hippocampus, and nucleus accumbens in each hemisphere, we analyzed the associations between the volumes of these regions and the level of perceived social support using the same methods. There were no significant main effects or interactions related to the perceived social support factor for any region $(p>0.1)$.

\subsection{Shape analysis}

Local shape analysis revealed significant positive associations ( $p<0.05$, family-wise errorcorrected) with the perceived social support score in clusters in the left amygdala (Fig. 2a). According to the highest assignment probability of the cytoarchitectonic map (Amunts et al., 2005; Eickhoff et al., 2005), peaks were located at the superficial subregion (Fig. 2b, left; Table 2) and the laterobasal subregion (Fig. 2b, right; Table 2). When the Pearson's product-moment correlations between perpendicular distances at these superficial and laterobasal subregion foci and the volume of the left amygdala were calculated, both foci showed significant positive correlations ( $r s=0.54$ and 0.49 ; $p$ s $<0.001$ ), suggesting that the local shapes in these subregions contribute to the global volume of the left amygdala. In the right amygdala, no significant cluster was detected.

\section{Discussion}

Our global volume analysis results demonstrated that larger left amygdala was associated with a higher level of perceived social support. The result is consistent with previous evidence in animals and humans that this structure plays an important role in social function (e.g., Dicks et al., 1969). A previous functional MRI study (Hyde et al., 2011) has also suggested amygdala involvement in perceived social support. In contrast to our results, two previous structural MRI studies did not find any significant association between amygdala volume and perceived social support (Bickart et al., 2011; Che et al., 2014a). Methodological differences may account for the disparity in these results. Che et al. (2014a) assessed perceived social support using the same questionnaire as that used in the current study but performed voxel-based morphometry. Because the shape analysis we conducted reportedly detected volume changes more sensitively than did voxel-based morphometry in the subcortical structures (Nemmi et al., 2015), the analysis we conducted might be more suitable for investigating subcortical structures such as the amygdala. Bickart et al. (2011) conducted the 
delineation and volume measurement of the amygdala as did we but assessed perceived social support using a different questionnaire (Social Provisions Scale; Russell et al., 1984). The questionnaire was developed to assess a broad range of interpersonal functions related to health and included some components not strictly related to social support, such as opportunity for nurturance (i.e., the sense that others rely on one for their well-being) (Cutrona \& Russell, 1987). The scores of these questionnaires are positively correlated, but only with moderate strength (Rizwan \& Syed, 2010). Hence, we speculate that the relatively narrowly defined perceived social support we assessed in this study might be more strongly related to amygdala structure. To our knowledge, this is the first evidence suggesting that amygdala volume is associated with perceived social support.

Our results showed laterality, in that the amygdala in only the left hemisphere was associated with perceived social support. The results are in line with previous studies of hemispheric functional asymmetry in brain-damaged patients (Buck \& Duffy, 1980) and in participants after amobarbital injection (Ross, Homan, \& Buck, 1994). The results of these studies suggested that the left hemisphere modulates social emotions (e.g., joy by gaining the affection of others), whereas the right hemisphere modulates primary emotions (e.g., ecstasy). The involvement of the left amygdala in the subjective perception of social support is also in line with previous functional neuroimaging results indicating that the left amygdala is related to the processing of subjectively perceived stimuli, whereas the right amygdala is involved in the processing of unconscious stimuli (Morris, Öhman, \& Dolan, 1998). Based on these data, we speculate that the perception of social support is processed dominantly in the left amygdala because of its social and subjective nature.

In addition, our shape analysis revealed that the laterobasal and superficial subregions of the left amygdala were associated with perceived social support. This result is consistent with several lines of evidence. These include human neuroimaging studies that reported activation of these, but not the centromedial, subregions in response to social stimuli (e.g., Hurlemann et al., 2008), a human stereological study that detected structural problems only in these subregions in autistic individuals with impaired social interactions (Schumann \& Amaral, 2006), and comparative anatomical studies that revealed an volume increase in these, but not the centromedial, regions in evolution corresponding with social functioning in primates (e.g., Barger et al., 2014). Additionally, a previous single unit recording study in monkeys demonstrated that neurons activated by social stimuli, such as faces, were more often located in the laterobasal, compared with the centromedial, subregions of the amygdala (Nakamura, Mikami, \& Kubota, 1992). Studies in rodents showed that social relationship deprivation impaired neuronal structural plasticity and neurotransmission in the laterobasal (AgisBalboa et al., 2007) and superficial (Schiller, Jahkel, \& Oehler, 2006) subregions but not in the centromedial subregoin of the amygdala. Together with these data, we speculate that one important social function of these amygdala subregions is to implement perceived social support.

Our results revealing amygdala involvement in perceived social support have implications for mechanisms linking perceived social support with psychological well-being, which remain unknown. Researchers have proposed that there would be a link between perceived social support and psychological well-being (e.g., Lakey \& Orehek, 2011), specifically through buffering against the effects of stress (e.g., Cohen \& Wills, 1985). Regarding the amygdala, which we found to be 
Social support and amygdala structure 9

associated with perceived social support, several structural neuroimaging studies reported its contraction related to psychological ill-being (e.g., post-traumatic stress disorder; Morey et al., 2012). A multimodal structural and functional neuroimaging study reported that a lower amygdala volume was associated with higher amygdala activation during a stressor task (Gianaros et al., 2008). Another multimodal neuroimaging study also assessing participants' genotypes showed lower amygdala volume and higher amygdala activity in response to negative emotional expressions in patients with genotypes associated with reduced serotonin transporter efficacy who have relatively high risks of psychological ill-being (Pezawas et al., 2005). Collectively, these data suggest the possibility that a lower level of perceived social support is linked to psychological ill-being via reduced structure, altered neurotransmitter signaling, and heightened activity in the amygdala, which is specifically relevant during stressful events.

Our results also provide implications for psychiatric disorders involving social impairments, such as ASD. Individuals with ASD are characterized primarily by deficient social interactions (American Psychiatric Association, 2013). Several previous studies have reported that individuals with ASD, compared with typically developing individuals, have a lower quality of life in the social domain in the World Health Organization Quality of Life-brief version (WHOQOL Group, 1988), which includes items asking about perceived social support (Lin, 2014; Jennes-Coussens, Magill-Evans, \& Koning, 2006; Kamio, Inada, \& Koyama, 2013; Kamp-Becker, Schroder, Remschmidt, \& Bachmann, 2010). Anatomical studies also reported reduced volumes (Nacewicz et al., 2006) and neuron numbers (Schumann et al., 2004) in the amygdala in individuals with ASD, specifically in the laterobasal and superficial subregions (Schumann \& Amaral, 2006). Together with these data, our data suggest that individuals with ASD may have lower perceived social support associated with smaller amygdala volumes. It would be interesting to investigate the issue in ASD and in other psychiatric disorders involving social impairment, such as schizophrenia and antisocial personality disorders.

Several limitations of this study should be acknowledged. First, because we used a cross-sectional design, a causal relationship between amygdala volume and perceived social support cannot be inferred. While one possibility is that individuals born with larger amygdala volumes perceive greater social support, another is that those who develop greater degrees of perceived social support have enlarged the amygdala volumes. Previous twin studies investigating perceived social support (Bergeman et al., 1990; Kessler et al., 1992; Raynor et al., 2002) and amygdala volume (den Braber et al., 2013; Kremen et al., 2010; Swagerman, Brouwer, de Geus, Hulshoff Pol, \& Boomsma, 2014) showed that both have high degrees of influence from both genetic and environmental factors, providing no conclusive support for either possibility. Future longitudinal studies are necessary to investigate any causal relationship between amygdala structure and perceived social support.

Second, because we investigated only perceived social support, it remains unknown whether amygdala structures could be associated specifically with perceived social support or generally with social functions related to perceived social support. Based on previous psychological evidence (e.g., Barrera, 1986), we can assume that perceived social support is mildly associated with enacted social support and social network properties. However, to further understand the neural substrate related to 
social support, it would be useful to measure and compare the relative strength of associations between these social support constructs and amygdala structure. This could be an important matter for future studies.

Third, because we focused on amygdala structure, other brain regions possibly associated with perceived social support were largely untested. Because the amygdala has widespread connections with other brain regions (Swanson \& Petrovich, 1998), it is plausible that the amygdala collaborates with other regions to implement perceived social support. Specifically, because a previous structural MRI study reported a positive association between the posterior cingulate cortex volume and perceived social support, scored using the same questionnaire as used in this study (Che et al., 2014a), the amygdala and posterior cingulate cortex may consist of a functional network associated with perceived social support. Consistent with this, several previous studies measuring resting-state functional MRI reported functional connectivity between these regions (e.g., Gabard-Durnam et al., 2014; Veer et al., 2011). Future studies analyzing structural and/or functional networks associated with perceived social support (e.g., Che et al., 2014b) would be promising with regard to interest in the amygdala.

Finally, this study had several technical limitations. For example, although the delineation method we used in this study (Patenaude et al., 2011) has several advantages, such as automatic and efficient delineation based on the knowledge of manually segmented images, it also has some limitations, such as difficulty in defining the anterior border of the amygdala, even using manual segmentation (cf. Brierley, Shaw, \& David, 2002). The use of a probabilistic cytoarchitectonic map in this study (Amunts et al., 2005; Eickhoff et al., 2005), which is currently the most widely used map of the amygdala subregions in neuroimaging studies, also has some limitations inherent in the spatial normalization of individual brains into MNI space (cf. Entis, Doerga, Barrett, \& Dickerson, 2012). Furthermore, it remains unclear which cellular mechanisms lead to macroscopic structural changes in the amygdala (cf. Zatorre, Fields, \& Johansen-Berg, 2012). Further technical improvements (e.g., ultra-high field MRI; Solano-Castiella et al., 2011) are needed to investigate amygdala structure and its association with perceived social support.

\section{Acknowledgments}

The authors thank Kazusa Minemoto, Akemi Inoue, and Emi Yokoyama for their technical support and our anonymous reviewers for their helpful advice. This study was supported by funds from the Japan Society for the Promotion of Science Funding Program for Next Generation World-Leading Researchers (LZ008). The authors declare no competing financial or other interests.

\section{References}

Adolphs, R. (2010). What does the amygdala contribute to social cognition? Annals of the New York Academy of Sciences, 1191, 42-61.

Agis-Balboa, R. C., Pinna, G., Pibiri, F., Kadriu, B., Costa, E., \& Guidotti, A. (2007). Downregulation of neurosteroid biosynthesis in corticolimbic circuits mediates social isolation-induced 
Social support and amygdala structure 11

behavior in mice. Proceedings of the National Academy of Sciences of the United States of America, 104, 18736-18741.

Amaral, D. G. (2003). The amygdala, social behavior, and danger detection. Annals of the New York Academy of Sciences, 1000, 337-347.

American Psychiatric Association (2013). Diagnostic and Statistical Manual of Mental Disorders: DSM-5 (5th ed.). American Psychiatric Association: Arlington, VA.

Amunts, K., Kedo, O., Kindler, M., Pieperhoff, P., Mohlberg, H., Shah, N. J., et al. (2005). Cytoarchitectonic mapping of the human amygdala, hippocampal region and entorhinal cortex: Intersubject variability and probability maps. Anatomy and Embryology, 210, 343-352.

Barrera, M. (1986). Distinctions between social support concepts, measures, and models. American Journal of Community Psychology, 14, 413-445.

Barger, N., Hanson, K. L., Teffer, K., Schenker-Ahmed, N. M., \& Semendeferi, K. (2014). Evidence for evolutionary specialization in human limbic structures. Frontiers in Human Neuroscience, 8, 277.

Barton, R. A., \& Aggleton, J. P. (2000). Primate evolution and the amygdala. In: J. P. Aggleton (ed.). The amygdala: A functional analysis. Oxford University Press, New York. p. 479-508.

Bergeman, C. S., Plomin, R., Pedersen, N. L., McClearn, G. E., \& Nesselroade, J. R. (1990). Genetic and environmental influences on social support: The Swedish Adoption/Twin Study of Aging. Journal of Gerontology, 45, 101-106.

Bickart, K. C., Wright, C. I., Dautoff, R. J., Dickerson, B. C., \& Barrett, L. F. (2011). Amygdala volume and social network size in humans. Nature Neuroscience, 14, 163-164.

Brierley, B., Shaw, P., \& David, A. S. (2002). The human amygdala: a systematic review and metaanalysis of volumetric magnetic resonance imaging. Brain Research. Brain Research Reviews, 39, 84-105.

Buck, R., \& Duffy, R. J. (1980). Nonverbal communication of affect in brain-damaged patients. Cortex, 16, 351-362.

Che, X., Wei, D., Li, W., Li, H., Qiao, L., Qiu, J., et al. (2014a). The correlation between gray matter volume and perceived social support: A voxel-based morphometry study. Social Neuroscience, 9, 152-159.

Che, X., Zhang, Q., Zhao, J., Wei, D., Li, B., Guo, Y., et al. (2014b). Synchronous activation within the default mode network correlates with perceived social support. Neuropsychologia, 63, 26-33.

Cohen, S., \& Wills, T. A. (1985). Stress, social support, and the buffering hypothesis. Psychological Bulletin, 98, 310-357.

Cook, R D. Weisberg, S. (1982). Residuals and influence in regression. Chapman \& Hall: New York. 
Social support and amygdala structure 12

Cummins, R. A. (2014). Measuring happiness and subjective well-being. In: S. David, I. Boniwell, \& A. Conley Ayers (eds.). Oxford handbook of happiness. Oxford University Press: Oxford. p. 185200.

Cutrona, C. E., \& Russell, D. W. (1987). The provisions of social relationships and adaptation to stress. Advances in Personal Relationships, 1, 37-67.

den Braber, A., Bohlken, M. M., Brouwer, R. M., van 't Ent, D., Kanai, R., Kahn, R. S., et al. (2013). Heritability of subcortical brain measures: A perspective for future genome-wide association studies. Neuroimage, 83, 98-102.

Dicks, D., Myers, R. E., \& Kling, A. (1969).Uncus and amiygdala lesions: effects on social behavior in the free-ranging rhesus monkey. Science, 165, 69-71.

Diener, E., \& Fujita, F. (1995). Resources, personal strivings, and subjective well-being: A nomothetic and idiographic approach. Journal of Personality and Social Psychology, 68, 926-935.

Eickhoff, S. B., Stephan, K. E., Mohlberg, H., Grefkes, C., Fink, G. R., Amunts, K., et al. (2005). A new SPM toolbox for combining probabilistic cytoarchitectonic maps and functional imaging data. NeuroImage, 25, 1325-1335.

Entis, J. J., Doerga, P., Barrett, L. F., \& Dickerson, B. C. (2012). A reliable protocol for the manual segmentation of the human amygdala and its subregions using ultra-high resolution MRI. Neuroimage, 60, 1226-1235.

Gabard-Durnam, L. J., Flannery, J., Goff, B., Gee, D. G., Humphreys, K. L., Telzer, E., et al. (2014). The development of human amygdala functional connectivity at rest from 4 to 23 years: A crosssectional study. Neuroimage, 95, 193-207.

Gianaros, P. J., Sheu, L. K., Matthews, K. A., Jennings, J. R., Manuck, S. B., \& Hariri, A. R. (2008). Individual differences in stressor-evoked blood pressure reactivity vary with activation, volume, and functional connectivity of the amygdala. Journal of Neuroscience, 28, 990-999.

Goossens, L., Kukolja, J., Onur, O. A., Fink, G. R., Maier, W., Griez, E., et al. (2009). Selective processing of social stimuli in the superficial amygdala. Human Brain Mapping, 30, 3332-3338.

Haber, M. G., Cohen, J. L., Lucas, T., \& Baltes, B. B. (2007). The relationship between self-reported received and perceived social support: A meta-analytic review. American Psychological Association Division of Community Psychology, 39, 133-144.

Heimer, L., de Olmos, J. S., Alheid, G. F., Pearson, J., Sakamoto, N., Shinoda, K., et al. (1999). The human basal forebrain Part II. In: F. E. Bloom, A. Björklund, T. Hökfelt (eds.). The primate nervous system, Part III. Elsevier: Amsterdam. p. 57-226.

Hurlemann, R., Rehme, A. K., Diessel, M., Kukolja, J., Maier, W., Walter, H., et al. (2008). Segregating intra-amygdalar responses to dynamic facial emotion with cytoarchitectonic maximum probability maps. Journal of Neuroscience Methods, 172, 13-20. 
Hyde, L. W., Gorka, A., Manuck, S. B., \& Hariri, A. R. (2011). Perceived social support moderates the link between threat-related amygdala reactivity and trait anxiety. Neuropsychologia, 49, 651656.

Iwasa, H., Gondo, Y., Masui, Y., Inagaki, H., Kawaai, T., Otsuka, R., et al. (2007). Nihongoban "Multidimensional Scale of Perceived Social Support” no shinraisei narabini datousei: Tyukonensya wo taisyou tosita kentou. Kousei no Shihyo, 54, 26-33.

Jennes-Coussens, M., Magill-Evans, J., \& Koning, C. (2006). The quality of life of young men with Asperger syndrome: A brief report. Autism, 10, 403-414.

Kamio, Y., Inada, N., \& Koyama, T. (2013). A nationwide survey on quality of life and associated factors of adults with high-functioning autism spectrum disorders. Autism, 17, 15-26.

Kamp-Becker, I., Schroder, J., Remschmidt, H., \& Bachmann, C. J. (2010). Health-related quality of life in adolescents and young adults with high functioning autism-spectrum disorder. Psychosocial Medicine, 7, 1-10.

Kanai, R., Bahrami, B., Roylance, R., \& Rees, G. (2012). Online social network size is reflected in human brain structure. Proceedings Biological Sciences, 279, 1327-1334.

Kessler, R. C., Kendler, K. S., Heath, A., Neale, M. C., \& Eaves, L. J. (1992). Social support, depressed mood, and adjustment to stress: A genetic epidemiologic investigation. Journal of Personality and Social Psychology, 62, 257-272.

Kling, A. S., \& Brothers, L. A. (1992). The amygdala and social behavior. In: J. P. Aggleton (ed.). The amygdala: Neurobiological aspects of emotion, memory, and mental dysfunction. Wiley-Liss: New York. p. 353-377.

Kremen, W. S., Prom-Wormley, E., Panizzon, M. S., Eyler, L. T., Fischl, B., Neale, M. C., et al. (2010). Genetic and environmental influences on the size of specific brain regions in midlife: the VETSA MRI study. Neuroimage, 49, 1213-1223.

Lakey, B. (2014). Perceived social support and happiness: The role of personality and relatoinal processes. In: S. David, I. Boniwell, A. Conley Ayers (eds.). Oxford handbook of happiness. Oxford University Press: Oxford. p. 847-859.

Lakey, B., \& Lutz, C. J. (1996). Social support and preventive and therapeutic interventions. In: G.R. Pierce, B.R. Sarason, I.G. Sarason (eds.). Handbook of social support and the family. New York: Plenum. p. 435-465.

Lakey, B., \& Orehek, E. (2011). Relational regulation theory: A new approach to explain the link between perceived social support and mental health. Psychological Review, 118, 482-495.

Lin, L Y. (2014). Quality of life of Taiwanese adults with autism spectrum disorder. PLoS One, 9, e109567. 
Social support and amygdala structure 14

López, M., \& Cooper, L. (2011). Social support measure review. Final report. First 5 LA: Los Angeles.

McCrae, R. R., \& Costa, P. T. Jr. (1987). Validation of the five-factor model of personality across instruments and observers. Journal of Personality and Social Psychology, 52, 81-90.

McDonald, A. J. (1998). Cortical pathways to the mammalian amygdala. Progress in Neurobiology, 55, 257-332.

Morey, R. A., Gold, A. L., LaBar, K. S., Beall, S. K., Brown, V. M., Haswell, C. C., et al. (2012). Amygdala volume changes in posttraumatic stress disorder in a large case-controlled veterans group. Archives of General Psychiatry, 69, 1169-1178.

Morris, J. S., Öhman, A., \& Dolan, R. J. (1998). Conscious and unconscious emotional learning in the human amygdala. Nature, 393, 467-470.

Nacewicz, B. M., Dalton, K. M., Johnstone, T., Long, M. T., McAuliff, E. M., Oakes, T. R., et al. (2006). Amygdala volume and nonverbal social impairment in adolescent and adult males with autism. Archives of General Psychiatry, 63, 1417-1428.

Nakamura, K., Mikami, A., \& Kubota, K. (1992). Activity of single neurons in the monkey amygdala during performance of a visual discrimination task. Journal of Neurophysiology, 67, 1447-1463.

Nemmi, F., Sabatini, U., Rascol, O., \& Peran, P. (2015). Parkinson’s disease and local atrophy in subcortical nuclei: Insight from shape analysis. Neurobiology of Aging, 36, 424-433.

Oldfield, R. C. (1971). The assessment and analysis of handedness: the Edinburgh inventory. Neuropsychologia, 9, 97-113.

Oswald, A. J., \& Wu, S. (2010). Objective confirmation of subjective measures of human well-being: Evidence from the U.S.A. Science, 327, 576-579.

Pardini, D. A., Raine, A., Erickson, K., \& Loeber, R. (2014). Lower amygdala volume in men is associated with childhood aggression, early psychopathic traits, and future violence. Biological Psychiatry, 75, 73-80.

Patenaude, B. (2007). Bayesian statistical models of shape and appearance for subcortical brain segmentation. University of Oxford.(http://ethos.bl.uk/OrderDetails.do?uin=uk.bl.ethos.491674).

Patenaude, B., Smith, S. M., Kennedy, D. N., \& Jenkinson, M. (2011). A Bayesian model of shape and appearance for subcortical brain segmentation. Neuroimage, 56, 907-922.

Pezawas, L., Meyer-Lindenberg, A., Drabant, E. M., Verchinski, B. A., Munoz, K. E., Kolachana, B. S., et al. (2005). 5-HTTLPR polymorphism impacts human cingulate-amygdala interactions: A genetic susceptibility mechanism for depression. Nature Neuroscience, 8, 828-834. 
Raynor, D. A., Pogue-Geile, M. F., Kamarck, T. W., McCaffery, J. M., \& Manuck, S. B. (2002). Covariation of psychosocial characteristics associated with cardiovascular disease: Genetic and environmental influences. Psychosomatic Medicine, 64, 191-203.

Rizwan, M., \& Syed, N. (2010). Urdu translation and psychometric properties of social provision scale. International Journal of Educational and Psychological Assessment, 4, 33-47.

Russell, D., Cutrona, C. E., Rose, J., \& Yurko, K. (1984). Social and emotional loneliness: An examination of Weiss's typology of loneliness. Journal of Personality and Social Psychology, 46, 1313-1321.

Ross, E. D., Homan, R. W., \& Buck, R. (1994). Differential hemispheric lateralization of primary and social emotions. Neuropsychiatry, Neuropsychology, and Behavioral Neurology, 7, 1-19.

Sato, W., Kubota, Y., Kochiyama, T., Uono, S., Yoshimura, S., Sawada, R., et al. (2014). Increased putamen volume in adults with autism spectrum disorder. Frontiers in Human Neuroscience, 8, 957.

Schiller, L., Jahkel, M., \& Oehler, J. (2006). The influence of sex and social isolation housing on preand postsynaptic 5-HT1A receptors. Brain Research, 1103, 76-87.

Schumann, C. M., \& Amaral, D. G. (2006). Stereological analysis of amygdala neuron number in autism. Journal of Neuroscience, 26, 7674-7679.

Schumann, C. M., Hamstra, J., Goodlin-Jones, B. L., Lotspeich, L. J., Kwon, H., Buonocore, M. H., et al. (2004). The amygdala is enlarged in children but not adolescents with autism; the hippocampus is enlarged at all ages. Journal of Neuroscience, 24, 6392-6401.

Shimonaka, Y., Nakazato, K., Gondo, Y., \& Takayama, M. (1999). Revised NEO-Personality Inventory (NEO-PI-R) and NEO Five-Factor Inventory (NEO-FFI) manual for the Japanese version. Tokyo Shinri: Tokyo.

Swagerman, S. C., Brouwer, R. M., de Geus, E. J., Hulshoff Pol, H. E., \& Boomsma, D I. (2014). Development and heritability of subcortical brain volumes at ages 9 and 12. Genes, Brain, and Behavior, 13, 733-742.

Sheehan, D. V., Lecrubier, Y., Sheehan, K. H., Amorim, P., Janavs, J., Weiller, E., et al. (1998). The Mini-International Neuropsychiatric Interview (M.I.N.I.): The development and validation of a structured diagnostic psychiatric interview for DSM-IV and ICD-10. Journal of Clinical Psychiatry, 59, 22-33.

Smith, S. M., \& Nichols, T. E. (2009). Threshold-free cluster enhancement: addressing problems of smoothing, threshold dependence and localisation in cluster inference. Neuroimage, 44, 83-98.

Solano-Castiella, E., Schäfer, A., Reimer, E., Türke, E., Pröger, T., Lohmann, G., et al. (2011). Parcellation of human amygdala in vivo using ultra high field structural MRI. Neuroimage, 58, 741-748. 
Social support and amygdala structure 16

Stephan, H., \& Andy, O. J. (1977). Quantitative comparison of the amygdala in insectivores and primates. Acta Anatomica, 98, 130-153.

Swanson, L. W., \& Petrovich, G. D. (1998). What is the amygdala? Trends in Neurosciences, 21, 323-331.

Uchino, B. N. (2009). Understanding the links between social support and physical health. A lifespan perspective with emphasis on the separability of perceived and received support.

Perspectives on Psychological Science, 4, 236-255.

Veer, I. M., Oei, N. Y., Spinhoven, P., van Buchem, M. A., Elzinga, B. M., \& Rombouts, S. A. (2011). Beyond acute social stress: Increased functional connectivity between amygdala and cortical midline structures. Neuroimage, 57, 1534-1541.

Von Der Heide, R., Vyas, G., \& Olson, I. R. (2014). The social network-network: Size is predicted by brain structure and function in the amygdala and paralimbic regions. Social Cognitive and Affective Neuroscience, 9, 1962-1972.

WHOQOL Group (1988). Development of the World Health Organization WHOQOL-BREF quality of life assessment. Psychological Medicine, 28, 551-558.

Winkler, A. M., Ridgway, G. R., Webster, M. A., Smith, S. M., \& Nichols, T. E. (2014). Permutation inference for the general linear model. Neuroimage, 92, 381-397.

Zatorre, R. J., Fields, R. D., \& Johansen-Berg, H. (2012). Plasticity in gray and white: neuroimaging changes in brain structure during learning. Nature Neuroscience, 15, 528-536.

Zimet, G. D., Dahlem, N. W., Zimet, S. D., \& Farley, G. K. (1988). The multidimensional scale of perceived social support. Journal of Personality Assessment, 52, 30-41.

Zimet, G. D., Powell, S. S., Farley, G. K., Werkman, S., \& Berkoff, K. A. (1990). Psychometric characteristics of the multidimensional scale of perceived social support. Journal of Personality Assessment, 55, 610-617. 
Table 1. Demographic and psychological rating data and their correlations.

\begin{tabular}{|c|c|c|c|c|c|c|c|c|c|c|c|c|c|c|c|c|}
\hline & \multirow[t]{2}{*}{ Variable } & \multirow[t]{2}{*}{$M / n$} & \multirow[t]{2}{*}{$S D$} & \multicolumn{13}{|c|}{ Correlation } \\
\hline & & & & 1 & & $2^{\mathrm{a}}$ & & 3 & & 4 & 5 & & 6 & & 7 & 8 \\
\hline 1 & Perceived Socisl Support & 5.2 & 1.1 & & & & & & & & & & & & & \\
\hline 2 & Sex (female:male) & 23:26 & & 0.32 & $*$ & & & & & & & & & & & \\
\hline 3 & Age & 22.4 & 4.4 & 0.04 & & -0.04 & & & & & & & & & & \\
\hline 4 & Full-scale Intelligence Quotient & 121.2 & 8.6 & 0.09 & & -0.11 & & -0.02 & & & & & & & & \\
\hline 5 & Neuroticism & 28.9 & 7.0 & -0.09 & & 0.02 & & -0.40 & $*$ & -0.10 & & & & & & \\
\hline 6 & Extraversion & 26.5 & 6.6 & 0.12 & & 0.18 & & 0.20 & & 0.06 & -0.07 & & & & & \\
\hline 7 & Openness-to-experience & 32.4 & 6.0 & 0.18 & & 0.03 & & 0.26 & & 0.03 & -0.34 & $*$ & 0.12 & & & \\
\hline 8 & Agreeableness & 30.4 & 5.2 & -0.06 & & 0.32 & $*$ & 0.06 & & 0.07 & 0.02 & & 0.31 & $*$ & -0.08 & \\
\hline 9 & Conscientiousness & 24.0 & 7.1 & 0.06 & & 0.06 & & 0.06 & & -0.11 & -0.15 & & 0.32 & $*$ & 0.03 & 0.04 \\
\hline
\end{tabular}

${ }^{\text {a }}$ Sex is dummy-coded as female (1) or male (0).

Correlations represent Pearson's product-moment correlations.

$* p<.05$. 
Table 2. Results of local shape analysis for the left amygdala. Positive associations between amygdala subregions and perceived social support score are shown.

\begin{tabular}{|c|c|c|c|c|c|c|c|}
\hline \multirow[t]{2}{*}{ Brain region } & \multicolumn{3}{|c|}{ Coordinates (mm) } & \multirow[t]{2}{*}{$p$-value } & \multicolumn{3}{|c|}{ Assignment probability } \\
\hline & $x$ & $y$ & $z$ & & Laterobasal & Superficial & Centromedial \\
\hline Amygdala & -17 & -4 & -16 & 0.012 & 11 & 54 & 19 \\
\hline Amygdala & -17 & -3 & -22 & 0.020 & 58 & 33 & - \\
\hline
\end{tabular}

Coordinates are in Montreal Neurological Institute space.

p-values are family-wise error-corrected.

Assignment probability is derived from Anatomy Toolbox. 
Social support and amygdala structure 19
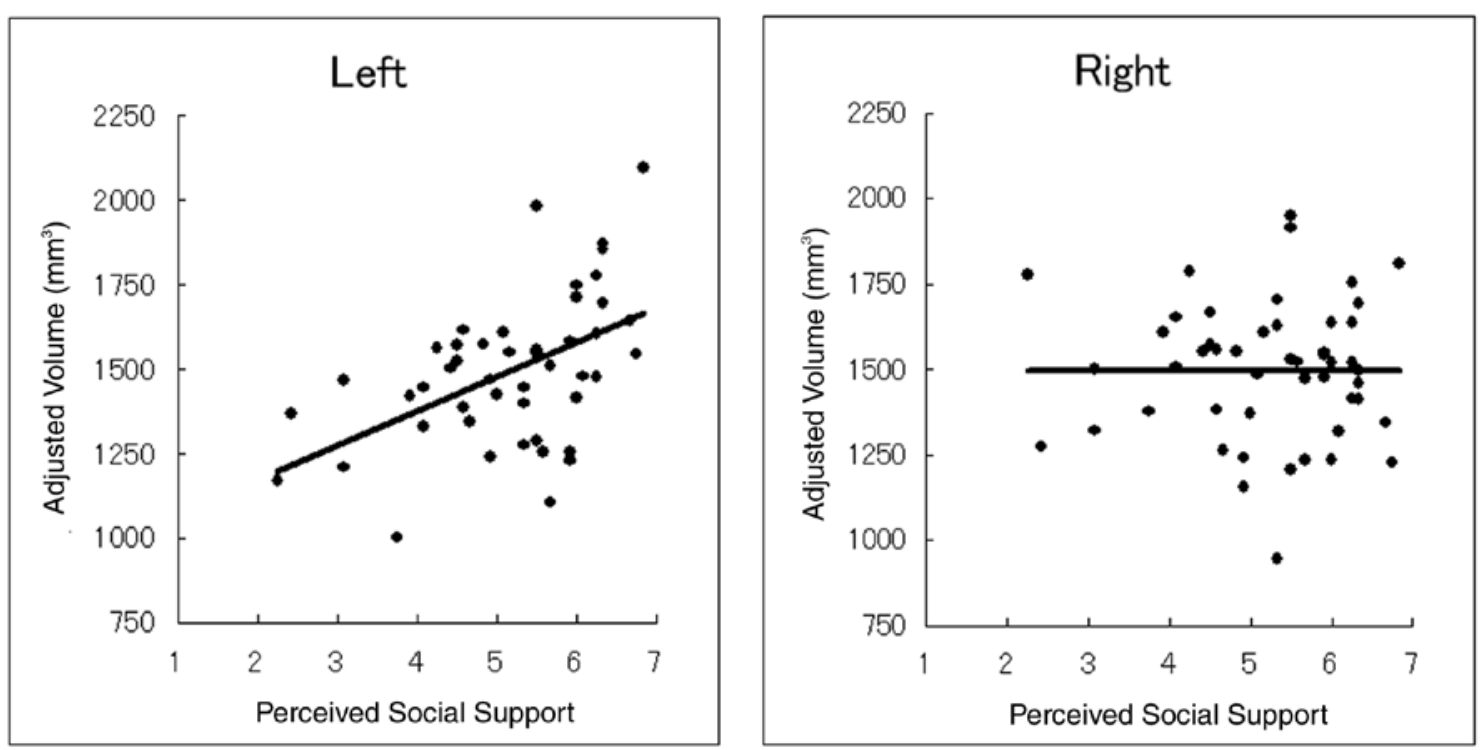

Figure 1. Scatter plots with regression lines of the adjusted volumes of the left $\left(R^{2}=0.27\right)$ and right $\left(R^{2}=0.19\right)$ amygdala as a function of perceived social support. 
a)
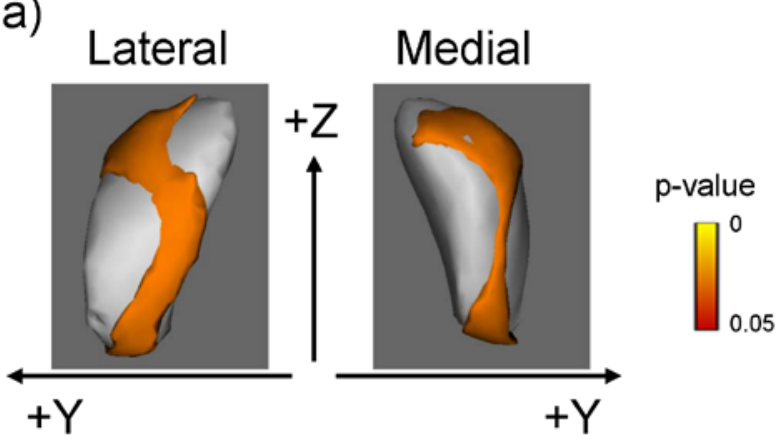

b)
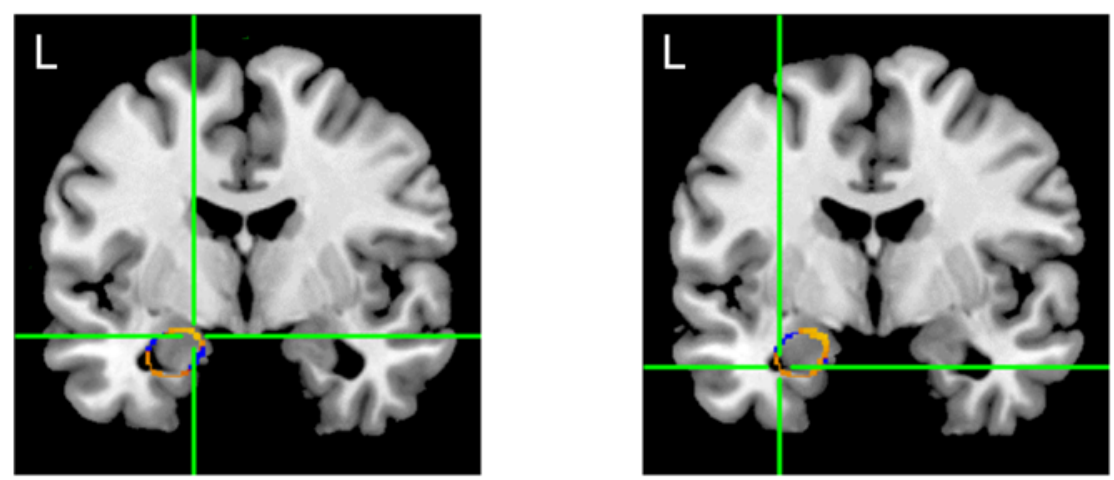

C)
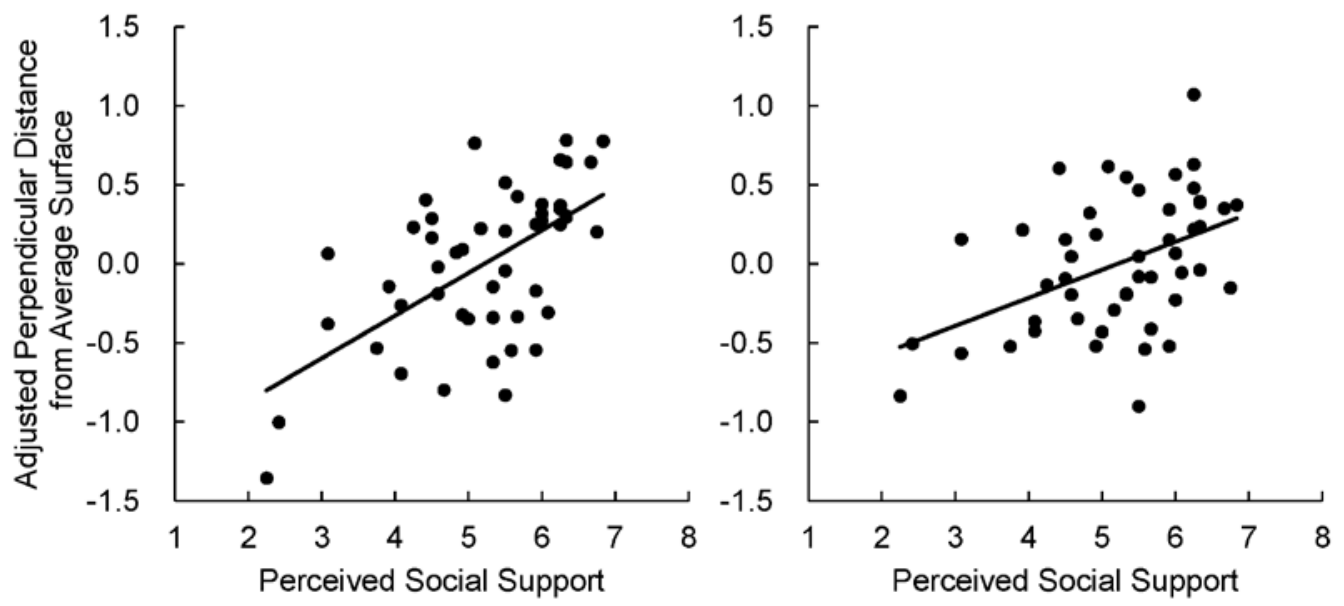

Figure 2. Results of local shape analysis for the left amygdala.

(a) Surface maps of the association between amygdala shape and perceived social support, in which gender, age, full-scale intelligence quotient, and the five domains of personality were regressed out as covariates of no interest. The yellow-red color reflects the $p$-value after cluster-level family-wise error correction for multiple comparisons.

(b) Axial sections in Montreal Neurological Institute (MNI) space showing the mean normalized amygdala shape in blue and the statistically significant cluster of association between vertex displacements and perceived social support in yellow-red ( $p$-values after cluster-level family-wise error correction for multiple comparisons). The green cross-hair indicates the local maximum at the 
superficial ( $x$-17 y-4 z-16; left) and laterobasal ( $x$-31 y-3z-26; right) subregions, assigned according to the highest probability using Anatomy Toolbox.

(c) Scatter plots with regression lines of the adjusted perpendicular distance from the average surface as a function of the perceived social support at the superficial (left; $R^{2}=0.36$ ) and laterobasal (right; $R^{2}=0.21$ ) subregions. 